\title{
Increase of HMGA1a protein methylation is a distinctive characteristic of leukaemic cells induced to undergo apoptosis
}

\author{
Cell Death and Differentiation (2003) 10, 386-389. doi: 10.1038/sj.cdd.4401184
}

\section{Dear Editor,}

HMGA1a, HMGA1b and HMGA2 are three small proteins (about 100 amino-acid residues) that constitute the family of nuclear phosphoproteins HMGA (previously known as HMGI, HMGY and HMGI-C, respectively). There is a special interest in these proteins because of their involvement in neoplastic transformation and gene expression regulation. ${ }^{1-4}$ HMGA1a and $H M G A 1 b$ are derived from the same gene by alternative splicing while HMGA2 is the product of a different but related gene. ${ }^{5,6}$ The sequence of HMGA proteins contains three positively charged regions called AT-hooks, which bind DNA at the minor groove of AT-rich stretches, and a C-terminus that, on the contrary, has a high negative charge because of several glutamic acid residues. ${ }^{3}$

In a previous paper, we investigated the change of the degree of phosphorylation of HMGA1a protein during apoptosis of leukaemic cells (HL60, K562, NB4 and U937). ${ }^{7}$ We found that this protein, constitutively phosphorylated in proliferating cells, undergoes hyperphosphorylation at the early stages of apoptosis which is later followed by a dephosphorylation process linked to the chromatin remodelling that accompanies the formation of the apoptotic bodies.

Here, we report a study on methylation of the HMGA1a protein that parallels the change in the degree of phosphorylation during apoptosis.

Methylation of HMGA1a protein in samples obtained from apoptotic leukaemic cells is observable in mass spectra as a peak having an additional mass of about $14 \mathrm{Da}$ more than the unmethylated form. ${ }^{7}$ In Figure $1 \mathrm{a}-\mathrm{d}$, apoptosis in $\mathrm{U} 937$ leukaemic cells was induced by chemical (etoposide or camptothecin) or viral (HSV-1) agents and protein samples obtained by perchloric acid (PCA) extraction were analysed by liquid chromatography/mass spectrometry (LC/MS) in order to distinguish all variously modified forms of HMGA1a protein. Results show that HMGA1a samples from control (Figure 1a) and apoptotic cells (Figure $1 \mathrm{~b}-\mathrm{d}$ ) comprise many species having a different degree of phosphorylation: $0 \mathrm{P}, 1 \mathrm{P}, 2 \mathrm{P}, 3 \mathrm{P}$ and $4 \mathrm{P} .{ }^{7}$ We emphasize that methylation of HMGA1a protein increases during apoptosis as suggested by the augmented presence of the right-hand peak having an additional mass of about $14 \mathrm{Da}(/ \mathrm{M})$. Moreover, Figure 1a-d shows that HMGA1a methylation, although related to the apoptotic process, is independent of the inducing agent, since samples obtained from cells induced to undergo apoptosis by three different procedures (etoposide, HSV-1 and camptothecin) contain high levels of methylated protein. In Figure 1e, the experimental mass values of the variously modified forms of
HMGA1a protein are reported being the theoretical mass value of human HMGA1a $11586.8 \mathrm{Da}$, according to the amino-acid sequence (i.e. 106 amino-acid residues) and assuming $\mathrm{N}$-terminal acetylation at the first serine. ${ }^{7}$ In order to demonstrate definitively that the additional mass value of about $14 \mathrm{Da}$ is the result of a methylation process, an inhibition experiment was carried out using adenosine dialdehyde (AdOx), which is an inhibitor of protein methylation in vivo. As shown in Figure $1 \mathrm{f}$ and $\mathrm{g}$, AdOx strongly reduced the level of methylation of HMGA1a protein demonstrating that the intensity increase of the 11760.3 and $11839.6 \mathrm{Da}$ peaks is actually because of the addition of a methyl group to the diphosphorylated $(11745.3 \pm 1 \mathrm{Da})$ and triphosphorylated (11825.8 $\pm 1 \mathrm{Da})$ forms of HMGA1a protein, respectively. Interestingly, the inhibition of methyltransferase activity does not prevent apoptosis as shown by the very similar DNA fragmentation patterns obtained from untreated and AdOxtreated apoptotic cells (Figure 1h, lanes $\mathrm{f}$ and $\mathrm{g}$, respectively). This means that methylation of HMGA1a protein is not one of the molecular events that causes apoptosis, but rather could be related to the cell death-induced chromatin remodelling.

Almost all HMGA1a in tumour cells is constituted by two forms containing, at the C-terminus of the molecule, two or three phosphorylated serines. ${ }^{7-9}$ We demonstrated that in leukaemic cells, at the early stages of apoptosis, there is an increase of phosphorylation, and hyperphosphorylated forms can be detected having up to five phosphates or more. ${ }^{7}$ As apoptosis proceeds, a dephosphorylation process starts and more or less overlaps with hyperphosphorylation depending on both the cell type and the method of apoptosis induction. Consequently, in a mass spectrum it is possible to observe hyperphosphorylated together with dephosphorylated HMGA1a forms. ${ }^{7}$ In the study reported here, we followed this process using U937 cells in which hyperphosphorylation is easily observable by the increase of the tri-phosphorylated form of HMGA1a protein in comparison to the diphosphorylated form, as shown in mass spectra of the time-course of Figure 2a-e. Here, in U937 cells treated with etoposide, the tri-phosphorylated form $(3 \mathrm{P}+3 \mathrm{P} / \mathrm{M})$ progressively increases as a result of the hyperphosphorylation process but, subsequently, there is a decrease in its amount. In addition, methylation progressively increases during the course of apoptosis, and continues to increase when the dephosphorylation of the tri-phosphorylated forms has begun. However, the progressive increase of dephosphorylation (note OP peak) parallels the methylation process, and it is possible that 

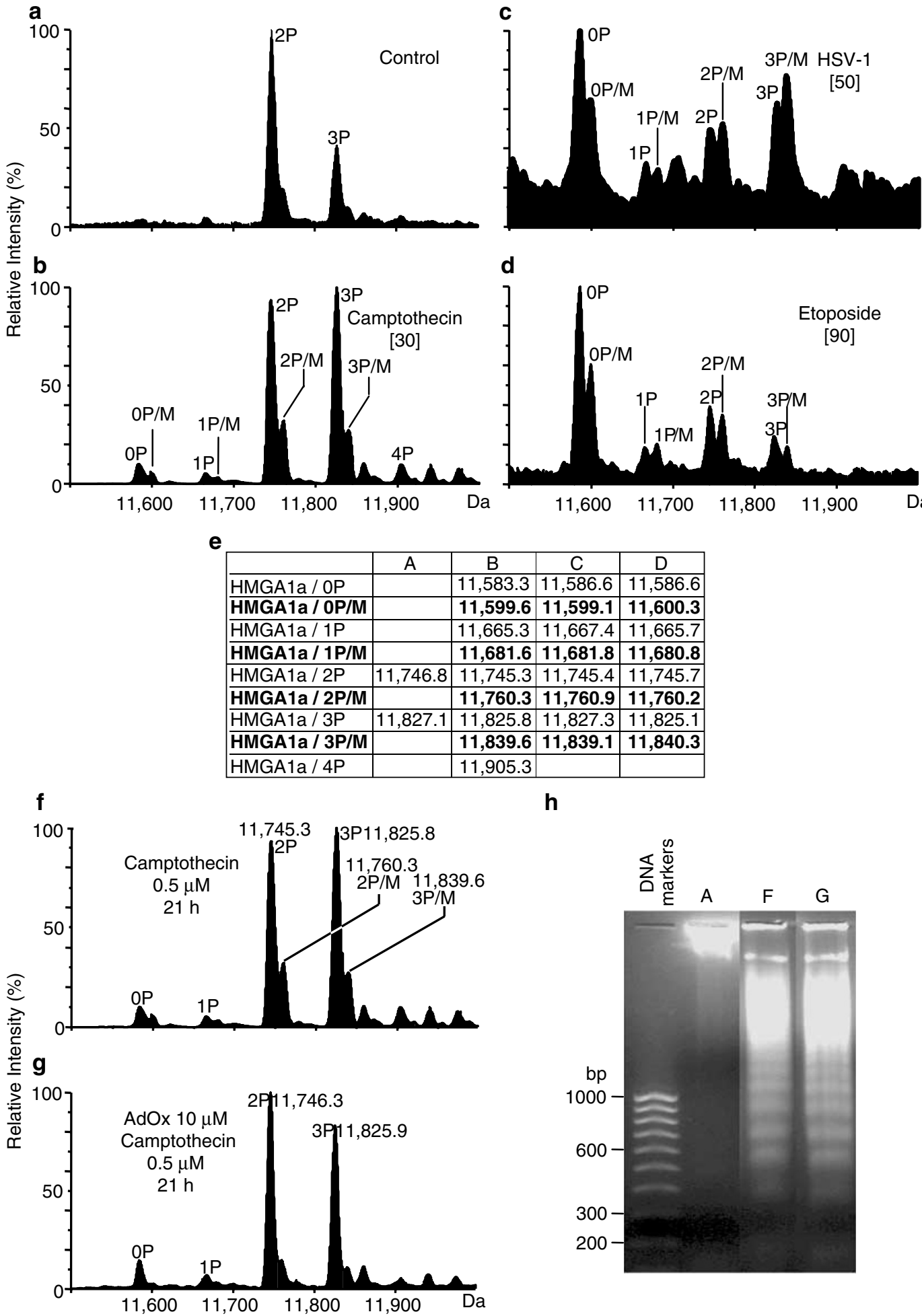

Figure 1 HMGA1a protein is methylated during apoptosis induced by different agents. HPLC-chromatographic peaks (not shown) of HMGA1a protein from U937 leukaemic cells were further analysed by mass spectrometry (PE SCIEX API 1) and results reported as reconstructed masses (Da). Each chromatographic HMGA1a peak contains more modified forms of the protein having different masses that permit identification of post-translational modifications. (a) Control U937 cells. (b) Camptothecin, (c) HSV-1 and (d) Etoposide-induced apoptotic U937 cells. OP, 1P, 2P, 3P and 4P indicate the number of phosphate groups (P) borne by HMGA1a protein while $\mathrm{M}$ indicate mono-methylation. Relative intensity of modified forms was evaluated as previously reported. ${ }^{7}$ In square brackets of (b)-(d) the percentage of apoptotic cells is shown. (e) Mass spectrometric values of the variously modified forms of HMGA1a protein of (a)-(d). Protein theoretical mass value obtained from amino-acid sequence and assuming N-terminal acetylation was $11586.8 \mathrm{Da}$. Mass values of methylated forms are shown in bold. (f) Camptothecin-induced U937 cells. (g) Camptothecin-induced and AdOx-treated U937 cells. (h) DNA fragmentation analysis of (a), (f) and (g) samples, respectively, on $2 \%$ agarose gel (ethidium bromide staining) 


\section{a}

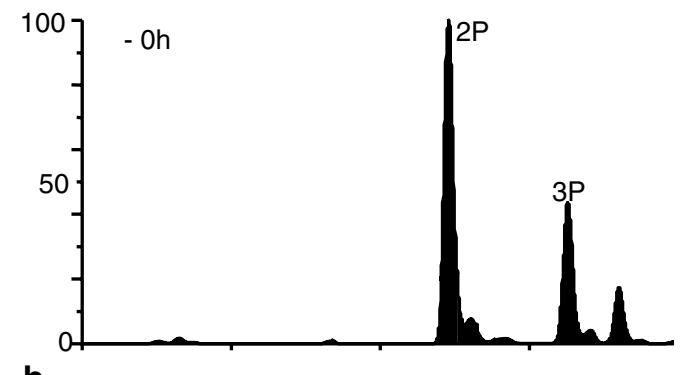

b
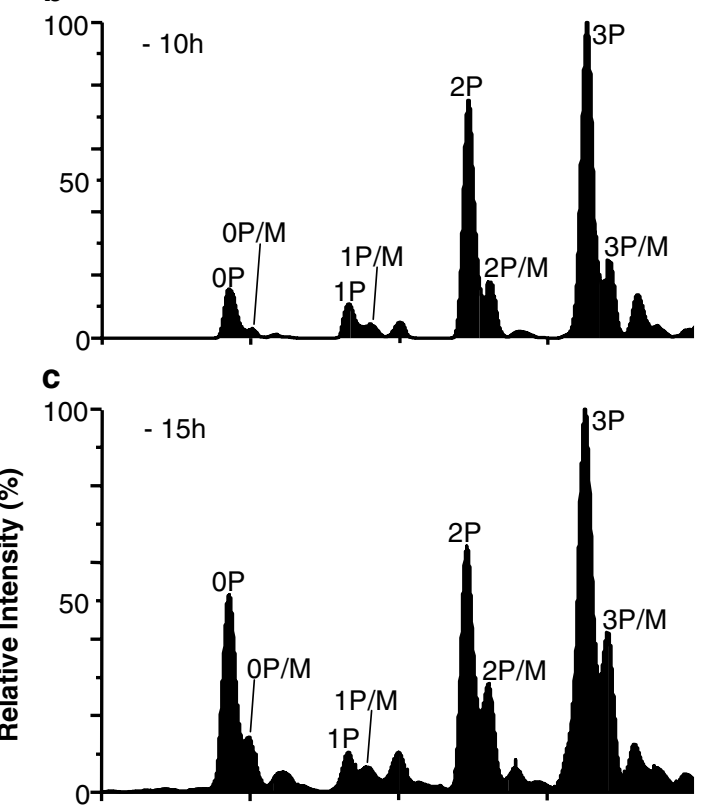

d

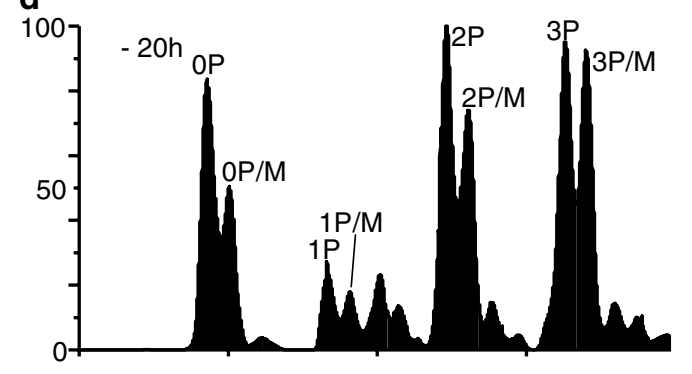$$
\text { e }
$$

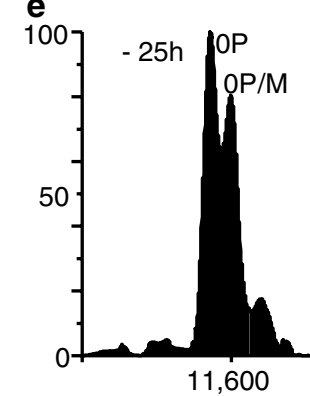

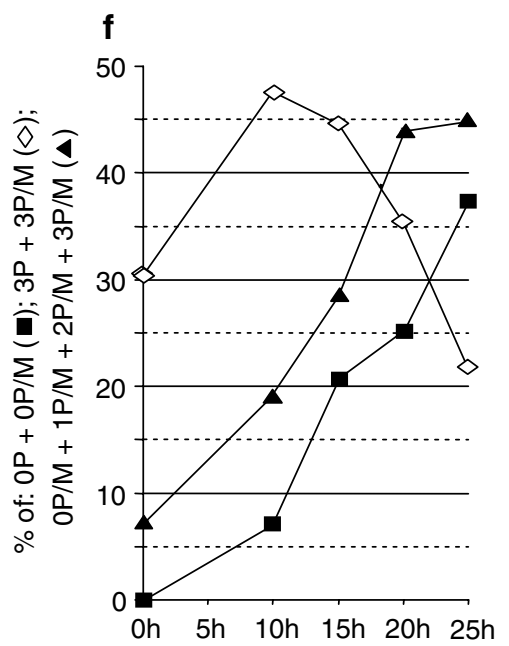

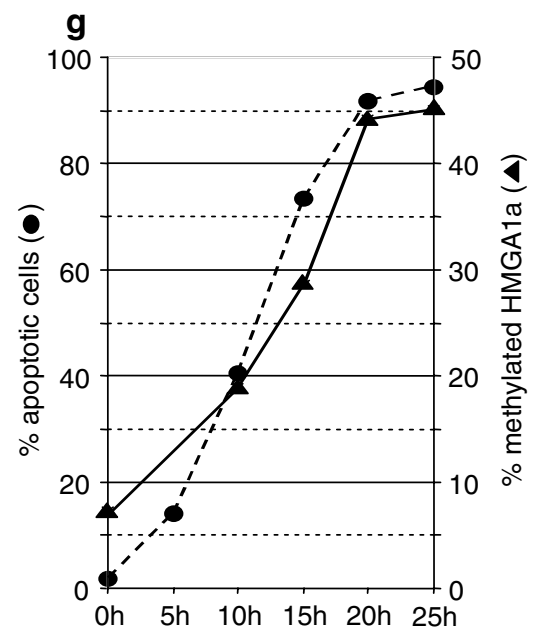

Oh 5 h 10 h 15 h 20 h $25 \mathrm{~h}$

h

Oh $10 \mathrm{~h} \quad 15 \mathrm{~h} \quad 20 \mathrm{~h} \quad 25 \mathrm{~h}$ markers

$\begin{array}{llllll}1 & 2 & 3 & 4 & 5 & 6\end{array}$

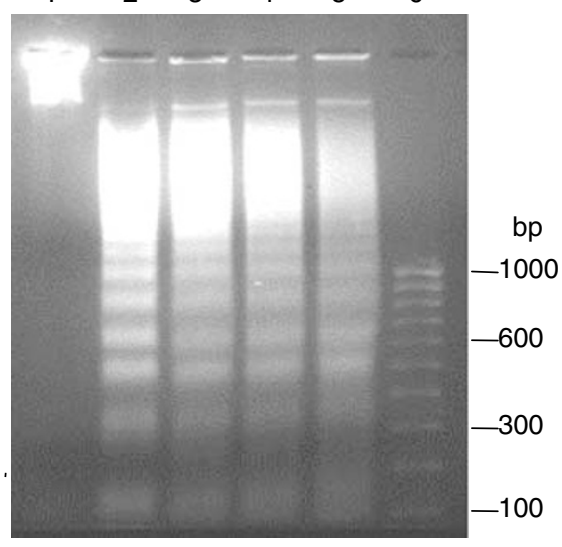

Figure 2 Time-course experiment demonstrates that HMGA1a dephosphorylation and methylation are parallel processes. U937 cells were induced to undergo apoptosis with etoposide for different times: $0,10,15,20$ and $25 \mathrm{~h}$ and mass spectra of HPLC-eluted HMGA1a peaks obtained (a)-(e). Percentage (f) of phosphorylated and/or methylated forms of HMGA1a is reported as follows: total unphosphorylated protein OP+OP/M $(\mathbf{D})$; total tri-phosphorylated protein $3 \mathrm{P}+3 \mathrm{P} / \mathrm{M}(\diamond)$; total methylated protein OP/M+1P/M+2P/M+3P/M ( $\mathbf{\Delta})$. (g) percentage of apoptotic U937 cells as in (a)-(e) measured by FACScan ( $)$; percentage of methylated HMGA1a protein as in (a)-(e) (A). (h) DNA fragmentation analysis of time-course samples $0,10,15,20$ and $25 \mathrm{~h}$ 
dephosphorylation and methylation of HMGA1a protein are two related post-translational modifications in this system. Figure $2 f$ stresses the time-course of the molecular events showing the percentage of all tri-phosphorylated forms (3P and $3 \mathrm{P} / \mathrm{M}$ ) in comparison to both the percentage of all unphosphorylated forms (OP and $\mathrm{OP} / \mathrm{M})$ and all monomethylated forms (OP/M, 1P/M, 2P/M and 3P/M). LC-MS analyses of Figure $2 \mathrm{a}-\mathrm{e}$ were complemented by the determination of the percentage of apoptotic cells by FACScan (Figure $2 \mathrm{~g}$ ) and electrophoretic DNA fragmentation analysis (Figure $2 \mathrm{~h}$ ) that provided additional information on the timecourse of etoposide-induced apoptosis. In fact, Figure $2 \mathrm{~g}$ shows that at $25 \mathrm{~h}$ about half of the HMGA1a protein is methylated when almost all cells are apoptotic. On the other hand, DNA analysis showed that a nucleosomal ladder is already observable at $10 \mathrm{~h}$, but most of the DNA is still formed by large fragments (approximately $20-50 \mathrm{kbp}$ ). The amount of high molecular weight DNA fragments is progressively reduced during apoptosis and parallels both the degree of dephosphorylation and methylation of HMGA1a protein.

A detailed study has been carried out using an HPLCpurified HMGA1a sample from HL-60 apoptotic cells in order to map the methylation site. LC-MS analysis of trypticdigested HMGA1a protein produced a peptide map (manuscript in preparation). Peptide overlapping sequences showed that arginine 25, located in the first AT-hook of HMGA1a protein, is methylated during apoptosis of leukaemic cells.

Finally, we verified if methylation of HMGA1a protein during apoptosis is only restricted to apoptotic leukaemic cells or could be considered a general feature of the apoptotic process. To this end, we carried out a study on other tumour cells of both human and murine origin. Preliminary results demonstrated that the increase of methylation of arginine 25 of HMGA1a protein could be considered a general property of apoptosis induction in tumour cells (data not shown).

In conclusion, these data show that (i) HMGA1a protein is heavily mono-methylated during apoptosis of different tumour cells, (ii) mono-methylation is at arginine 25 in the first AT- hook, (iii) methylation parallels the dephosphorylation process of the protein. Therefore, we propose that the coupled processes of methylation/dephosphorylation found for HMGA1a protein could be regarded in the general context of the combination of three modifications, that is, phosphorylation, acetylation and methylation that influence the formation of protein complexes in regulating chromatin remodelling and gene activity.

\section{Acknowledgments}

This work was supported by grants from Associazione Italiana per la Ricerca sul Cancro, Milano, Italy, ASI (N. I/R 058/01 and N. I/R 129/00), MIUR (PRIN, 2002), and Università di Trieste, Italy. RS is a postdoctoral fellow supported by FIRC, Milano, Italy.

\section{R Sgarra ${ }^{1}, F$ Diana $^{1}$, A Rustighi', G Manfioletti ${ }^{1}$ and $V$ Giancotti $^{*}, 1,2$ \\ 1 Dipartimento di Biochimica, Biofisica e Chimica delle Macromolecole, Università di Trieste, Trieste, Italy \\ 2 Dipartimento di Patologia e Medicina Sperimentale e Clinica, Università di Udine, Udine, Italy \\ * Corresponding author: V Giancotti, Current address: Dipartimento di Patologia e Medicina Sperimentale e Clinica, Università di Udine, P, le S. Maria della Misericordia, 33100 Udine, Italy; E-mail: giancot@bbcm.univ.trieste.it}

1. Giancotti V et al. (1985) Cancer Res. 45: 6051-6057.

2. Giancotti V et al. (1987) EMBO J. 6: 1981-1987.

3. Bustin M and Reeves R (1996) In Progress in Nucleic Acids Research and Molecular Biology, Cohn WE and Moldave K, eds (New York: Academic Press) vol 84 , pp. $35-100$

4. Jansen E et al. (1999) Gene Ther. Mol. Biol. 3: 387-395.

5. Manfioletti $G$ et al. (1991) Nucleic Acids Res. 19: 6793-6797.

6. Manfioletti G et al. (1995) Gene 167: 249-253.

7. Diana F et al. (2001) J. Biol. Chem. 276: 11354-11361.

8. Giancotti V et al. (1991) Eur. J. Biochem. 198: 211-216.

9. Ferranti $\mathrm{P}$ et al. (1992) J. Biol. Chem. 267: 22486-22489. 